\title{
CARACTERÍSTICAS FÍSICO-QUÍMICAS DO ANTIMONIATO DE MEGLUMINA EM DIFERENTES CONDIÇÕES DE ARMAZENAMENTO
}

\author{
Gustavo Adolfo Sierra Romero, Maria Regina Fernandes de Oliveira, \\ Dalmo Correia e Philip Davis Marsden
}

\begin{abstract}
No periodo de outubro de 1992 a julbo de 1995, realizaram-se mediçôes da osmolaridade e pH de ampolas pertencentes a um unico lote de meglumina antimoniato (Rbodia Farma Ltda, Säo Paulo, SP, Brasil. Lote 9206L-004) mantidos em três condiçöes de temperatura $\left(4^{\circ} \mathrm{C}, 37^{\circ} \mathrm{C}\right.$ e temperatura ambiente). Embora fossem observadas diferenças estatisticamente significativas na osmolaridade média das ampolas submetidas aos diferentes tratamentos, o numero limitado de mediçōes $e$ a variabilidade desta característica entre ampolas mantidas na mesma condição de temperatura, avaliadas no mesmo monento, não permitiram obter conclusões definitivas. Não foi demonstrada tariação significativa do pH nas mesmas condiçoes. Assumindo que a alteração nestes parâmetros poderia refletir mudanças na estrutura do antimonial pentavalente está indicada a realização de esperimentos melbor controlados que possam definir a relação existente entre as variáueis estudadas.
\end{abstract}

Palavras-chaves: Antimonial pentatalente. Osmolaridade. pH. Temperatura de armazenamento

Os antimoniais pentavalentes continuam sendo as drogas de primeira escolha para o tratamento das infecções produzidas por Leishmania ${ }^{12}$. Existem no mercado duas apresentações: o estibogluconato de sódio (pentostam $($ ) ) o antimoniato de $\mathrm{N}$-metilglucamina (glucantimeß) sendo esta última fabricada por Rhodia Farma Ltda, para o Ministério da Saúde do Brasil e distribuída pela Central de Medicamentos com o nome genérico de meglumina antimoniato em ampolas de $5 \mathrm{ml}$ contendo $425 \mathrm{mg}$ de $\mathrm{Sb}^{r}(85 \mathrm{mg} / \mathrm{ml})$, segundo as informações do fabricante.

Reconhece-se que estas drogas possuem um nivel de toxicidade importante $>8910$, embora menor que o observado com os antimoniais trivalentes ( $\left.\mathrm{Sb}^{\mathrm{II}}\right)$ e até hoje ignorase a sua estrutura química real ${ }^{10}$ e o mecanismo de ação só é parcialmente conhecido ${ }^{23}$. Recentemente tem-se demonstrado, no conteúdo das ampolas, a presença de antimonial trivalente $\left(\mathrm{Sb}^{\mathrm{II}}\right)$ e variabilidade na quantidade de $\mathrm{Sb}^{\text {vs } 6}$.

\footnotetext{
Núcleo de Medicina Tropical e Nutrição, Universidade de Brasília, Brasília, DF:

Endereço para correspondência: Dr. Gustavo Adolfo Sierra Romero. Núcleo de Medicina Tropical, Universidade de Brasília, Caixa postal 04671, Brasília, DF. 709-9970. Fax: (061) 273-2811 E-mail: gromero@guarany.cpd.unb.br Recebido para publicação em 30/10/95.
}

Durante a realizacão de estudos terapêuticos numa área endêmica de leishmaniose estabeleceu-se que a osmolaridade e a cor do antimonial utilizado é diferente para cada lote do medicamento (PD Marsden e GAS Romero: dados não publicados).

O objetivo do estudo foi determinar se existe variação na osmolaridade e o pH do medicamento armazenado em três condições de temperatura diferentes.

\section{MATERIAL E MÉTODOS}

Foram utilizadas 150 ampolas de meglumina antimoniato (Rhodia Farma Ltda, São Paulo, SP, Brasil. Lote 9206L-004) mantidas inicialmente a $4^{\circ} \mathrm{C}$ e posteriormente divididas em três grupos de 50 ampolas que foram colocados em três condições de termperatura: $4^{\circ} \mathrm{C}$ (Grupo 1), $37^{\circ} \mathrm{C}$ (Grupo 2) e a temperatura ambiente (Grupo 3). A temperatura média do ar em Brasília durante o período de outubro de 1993 a setembro de 1995 foi de $21,3^{\circ} \mathrm{C}$ (média mensal, intervalo de 18,1 a $23,7^{\circ} \mathrm{C}$ ) calculada diariamente pela fórmula: $\mathrm{T}=[\mathrm{T}(12 \mathrm{~h})+2 \mathrm{~T}(24 \mathrm{~h})+\mathrm{T}$. máxima $+\mathrm{T}$. mínima $] / 5$.

O medicamento manteve-se protegido da luz e todas as medições foram realizadas dentro do período de validade indicado pelo fabricante (60 meses). Foram realizadas 11 
Romero GAS, Olineira MRF, Correia Filho D, Marsden PD. Caracteristicas fisiso-químicas do antimoniato de meglumina em diferentes condiçóes de armazenamento. Revista da Sociedade Brasileira de Medicina Tropical 29:461-465, set-out, 1996.

mediçôes da osmolaridade e 8 medições do pH com o osmômetro de Fiske (Fiske Associates Inc. Oxbrige Mass, USA) e o pHmetro digital (Micronal B222, Brasil). Antes de cada medição os instrumentos foram calibrados adequadamente utilizando soluções padrão e a medição da osmolaridade foi realizada por um único observador durante toda a experiência.

\section{RESULTADOS}

As primeiras três medições da osmolaridade constituem a média da osmolaridade de três ampolas diferentes do grupo 1. A partir da quarta até a sétima medição, o valor da osmolaridade e o pH correspondem à medição em uma ampola de cada grupo e a partir da oitava até a décima primeira medição, os valores da osmolaridade e o pH correspondem à média das mediçôes em duas ampolas de cada grupo. A média das medições da osmolaridade dos três grupos, durante o período completo de acompanhamento, foi: Grupo $1=844,2 \mathrm{mosm} / 1$ (desvio padrão $=16,9)$, Grupo $2=859,4 \mathrm{mosm} / \mathrm{l}$ (desvio padrão $=19,6)$ e Grupo $3=857,4 \mathrm{mosm} / 1$ (desvio padrão = 17,9). Houve diferença estatisticamente significativa entre as médias dos grupos 1 e 2 $(\mathrm{t}=2,343 ; \mathrm{p}<0,025 ;$ Intervalo de Confiança $\left.95 \%\left(\mathrm{Cl}_{95 \%}\right)=[1,95 ; 28,41 \mathrm{mosm} / \mathrm{l}]\right)$ e as médias dos grupos 1 e $3\left(\mathrm{t}=2,1089 ; \mathrm{p}<0,025 ; \mathrm{CI}_{95 \%}=\right.$ $[0,42 ; 25,94 \mathrm{mosm} / 1])$. Não houve diferença entre as médias dos grupos 2 e $3(t=0,2607$; $\left.\mathrm{p}>0,10 ; \mathrm{CI}_{95 \%}=[-13,90 ; 17,90 \mathrm{mosm} / 1]\right)$ (Figura 1).

As médias das medições do $\mathrm{pH}$ foram: Grupo 1 =5,48, Grupo $2=5,56$ e Grupo 3=5,52. Não houve diferença estatisticamente significativa entre os grupos (análise de variância: $F=0,168, p=0,85$ ) ( Figura 2).

\section{DISCUSSÃO}

Até hoje não existem dados sobre a influência de fatores como a temperatura e a exposição à luz sobre as características físicoquímicas dos antimoniais pentavalentes e sobre a relação da osmolaridade e o pH com outras variáveis que possam refletir deterioração do medicamento em diferentes condições de armazenamento. A instabilidade dos antimoniais foi descrita por Brahmachari na Índia ${ }^{11} \mathrm{e}$ conhece-se que podem sofrer transformações importantes sendo a mais perigosa a redução ao $\mathrm{Sb}^{\text {II }}$ que constitui uma espécie química muito mais tóxica ${ }^{610}$. O presente trabalho mostra que a osmolaridade do medicamento poderia aumentar significativamente quando é mantido a temperaturas elevadas (Grupo 2) ou não controladas (Grupo 3), tendo como referência o medicamento mantido a $4^{\circ} \mathrm{C}$ (Grupo 1 ). Durante a realização deste trabalho foi evidente a variação no valor da osmolaridade entre ampolas de um mesmo grupo, medida

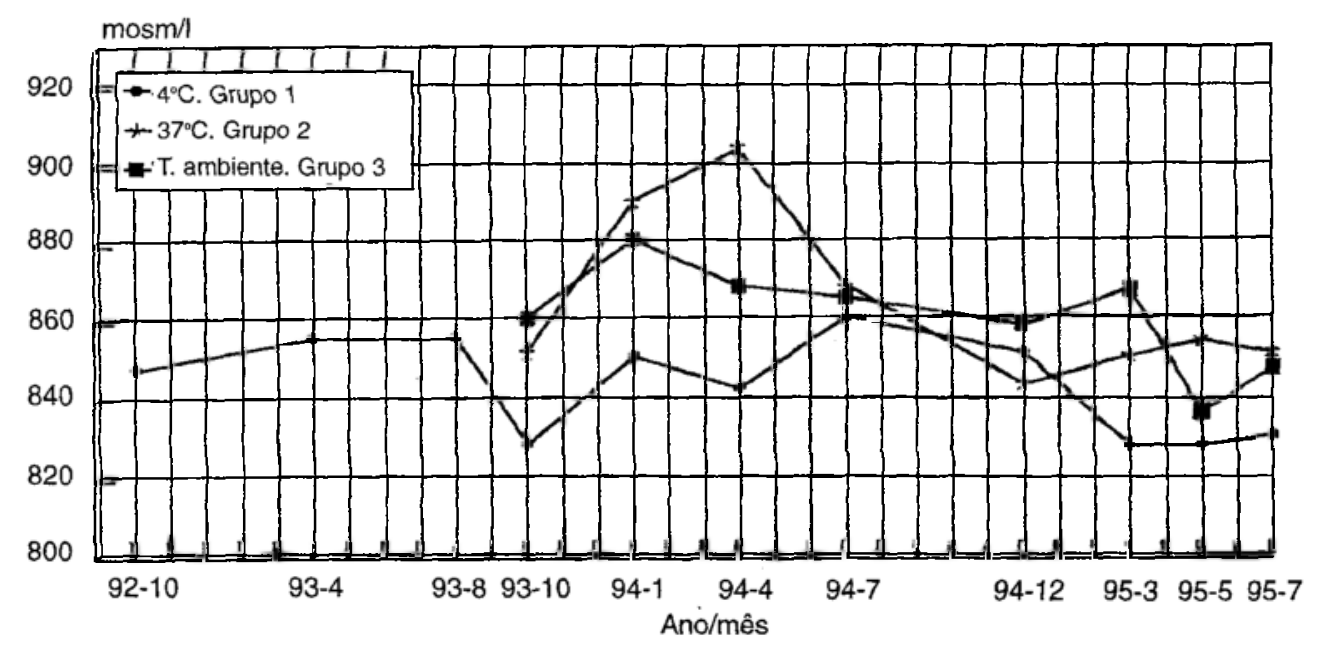

Figura 1 - Osmolaridade do antimoniato de meglumina armazenado em três condiçòes de temperatura. 
Romero GAS, Oliveira MRF, Correia Filbo D, Marsden PD. Características fisiso-quimicas do antimoniato de meglumina em diferentes condições de armazenamento. Revista da Sociedade Brasileira de Medicina Tropical 29:461-465, set-out, 1996.

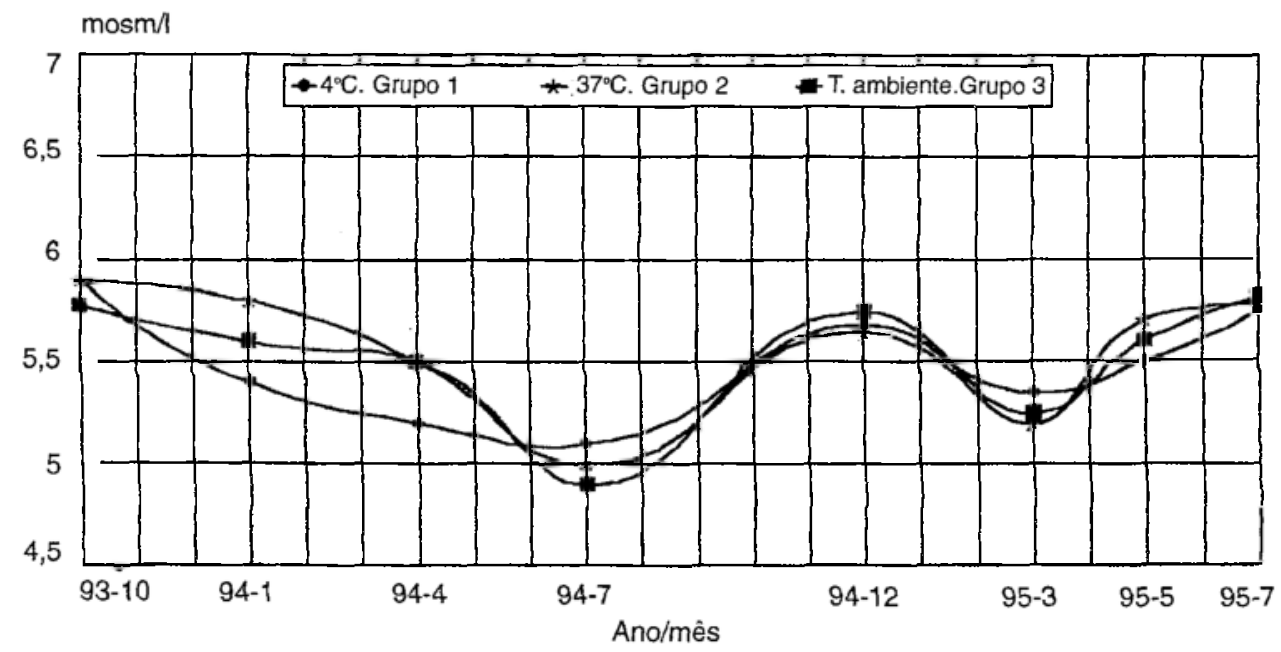

Figura 2 - pH do antimoniato de meglumina armazenado em três condiçōes de temperatura.

no mesmo momento, pelo que se poderiam atribuir as diferenças observadas, neste experimento, a essa variabilidade. Realizamos uma última avaliação em outubro de 1995. medindo a osmolaridade de 10 ampolas de cada grupo (Figura 3). A análise dos resultados desta última medição não confirmou as diferenças detectadas inicialmente, observando-

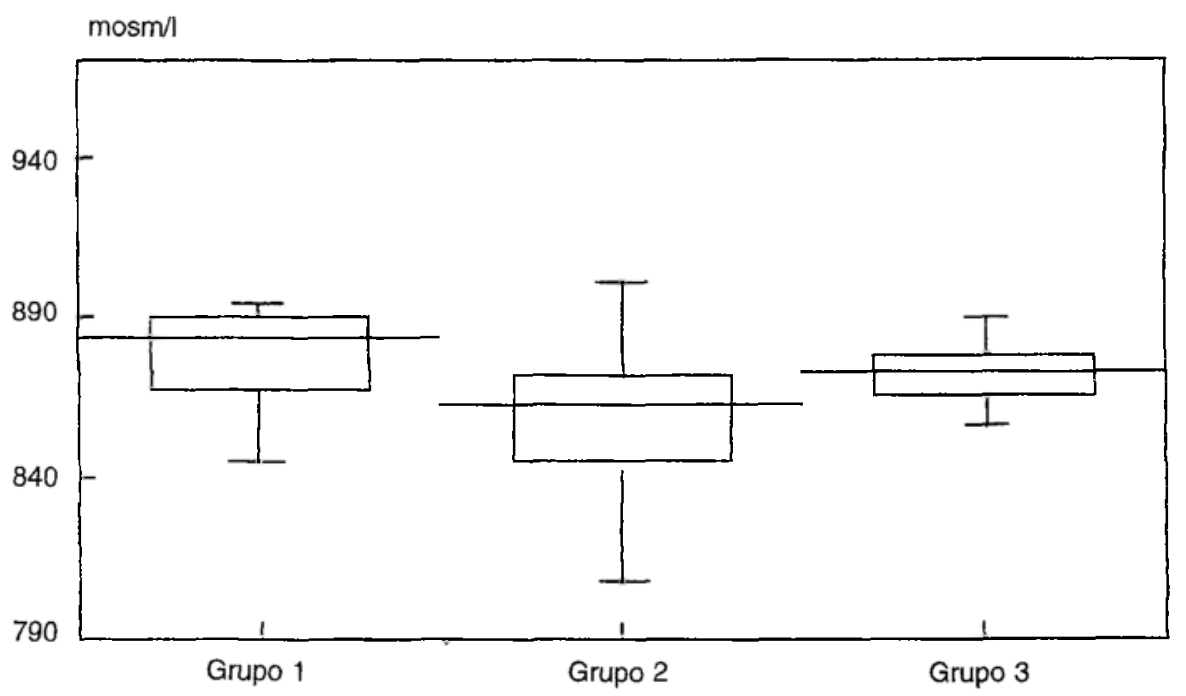

Figura 3 - Distribuição dos valores da osmolaridade medida ao final da experiência ao redor da mediana (barras borizontais. 
Romero GAS, Oliveira MRF, Correia Filbo D, Marsden PD. Caracteristicas fisiso-químicas do antimoniato de meglumina em diferentes condiçôes de armazenamento. Revista da Sociedade Brasileira de Medicina Tropical 29:461-465, set-out, 1996.

se como única diferença com significância estatística, que a osmolaridade no grupo 2 $\left(37^{\circ} \mathrm{C}\right)$ foi menor que a do grupo $1\left(4^{\circ} \mathrm{C}\right) .(\mathrm{t}=$ 2,$\left.218 ; \mathrm{p}<0,025 ; \mathrm{Cl}^{95 \%}=[1,09 ; 40,11 \mathrm{mosm} / \mathrm{l}]\right)$.

Analisando globalmente estes resultados é muito provável que as diferenças detectadas pelos testes estatísticos não sejam reais e que reflitam as variações existentes entre as ampolas independentemente da açâo de temperaturas diferentes. Os intervalos de confiança calculados para as diferenças com significância estatística têm limites muito amplos o que apoia a importância da variabilidade da osmolaridade entre ampolas de um mesmo grupo. Lamentavelmente, na fase inicial do experimento não foi considerada a variabilidade da osmolaridade entre ampolas de um mesmo lote o que impediu que se tivesse um controle adequado para fazer as comparações entre grupos. As diferenças de osmolaridade entre lotes do medicamento (PD Marsden, GAS Romero: dados não publicados) são muito maiores que as observadas entre os grupos de um mesmo lote em condiçōes de temperatura diferentes pelo que a medição desta variável não parece ser um método sensivel para avaliar a potencial deterioração do medicamento através do tempo. Considerando que as medições da osmolaridade e o pH sào fáceis de serem realizadas, poderiam ser úteis na padronização inicial do medicamento sempre que investigações posteriores demonstrassem que têm relação com a quantidade de $\mathrm{Sb}^{\mathrm{v}} \mathrm{e} / \mathrm{ou} \mathrm{Sb}^{\mathrm{mI}}$ no conteúdo das ampolas. Outro fator que deve ser estudado é a influência da exposição à luz de diferentes longitudes de onda sob condiçôes de temperatura controladas para acrescentar o conhecimento sobre fatores externos facilmente corrigiveis que poderiam contribuir a manter o medicamento em condições estáveis. Enquanto não se disponha de mais informação a respeito parece razoável continuar recomendando o armazenamento dos antimoniais a $4^{\circ} \mathrm{C}$ e protegidos da luz ${ }^{10}$.

\section{SUMMARY}

During the period October 1992 to July 1995 we measured the osmolarity and $p H$ of ampoules of meglumina antimoniato (glucantime $\left.{ }^{\circledR}\right)$ from lot 9206L-004 (manufacturated by Rhodia Farma Ltd, of São Paulo, SP, Brazil) mantained in tbree temperature conditions namely $4^{\circ} \mathrm{C}, 37 \circ \mathrm{C}$ and ambiental. Although we observed statistically significant differences in osmolarity between samples, the limited number of measivements and the variation of this property in ampoles mantained at the same temperature were obstacles to obtain definitive conclusions. Such a variation was not. found with $p H$. Assuming these parameters could reflect structural changes in the pentavalent antimony molecule, clearly furtber better controlled experiments are indicated.

Key-words: Pentavalent antimonial. Osmolarity. pH. Temperature of storage.

\section{AGRADECIMENTOS}

Agradecemos ao Prof. Isaac Roitmann pelas facilidades para medir o $\mathrm{pH}$, ao Sr. Fernando Vicente de Pádua pela sua dedicação na medição da osmolaridade e à Prof ${ }^{2}$ Ana Maria Vasconcelos pela revisão crítica da análise estatística.

\section{REFERÊNCIAS BIBLIOGRÁFICAS}

1. Berman JD. Chemotherapy for leishmaniasis: Biochemical mechanisms, clinical efficacy and future strategies. Reviews of Infectious Diseases 10:560-586,1988.

2. Berman JD, Gallalee JV, Best JM. Sodium stibogluconate (pentostam) inhibition of glucose catabolism via the glycolytic pathway, and fatty acid B-oxidation in Leishmania mexicana amastigotes. Biochemical Phatmacology 36:197201,1987.

3. Berman JD, Waddell D, Hanson BD. Biochemical mechanisms of antileishmanial activity of sodium stibogluconate. Antimicrobial Agents and Chemotherapy 27:916-920,1985.

4. Chulay JD, spencer HC, Mugambi M. Electrocardiographic changes during treatment of leishmaniasis with pentavalent antimony (sodium stibogluconate). The American Journal of Tropical Medicine and Hygiene 34:702-709, 1985.

5. Franco MA. Determinação de antimoniais $\left(\mathrm{Sb}^{\mathrm{IIt}} \mathrm{e}\right.$ $\mathrm{Sb}^{v}$ ) em fármacos. Tese de mestrado, Universidade de Brasília, Brasilia, DF, 1992.

6. Franco MA, Barbosa AC, Rath S, Dorea JG. Antimony oxidation states in antileishmanial drugs. The American Journal of Tropical Medicine and Hygiene 52:435-437,1995.

7. Gasser Jr RA, Magill AJ, Oster CN, Franke ED, Grogl LM, Berman JD. Pancreatitis induced by pentavalent antimonial agents during treatment of leishmaniasis. Clinical Infectious Diseases 18:83-90,1994. 
Romero GAS, Olineira MRF, Conreia Filho D, Marsden PD. Caracteristicas fisiso-químicas do antimoniato de meglumina em diferentes condições de armazenamento. Revista da Sociedade Brasileira de Medicina Tropical 29:461-465, set-out, 1996.

8. Hepburn NC. Thrombocytopenia complicating sodium stibogluconate therapy for cutaneous leishmaniasis. Transactions of the Royal Society of Tropical Medicine and Hygiene 87:691,1993.

9. Hepburn NC, Siddique I, Howie AF, Beckett GJ, Hayes PC. Hepatotoxicity of sodium stibogluconate therapy for American cutaneous leishmaniasis. Transactions of the Royal Society of Tropical Medicine and Hygiene 88:453-455,1994.

10. Marsden PD. Pentavalent antimonials: old drugs for new diseases. Revista da Sociedade Brasileira de Medicina Tropical 18:187-198,1985.
11. Marsden PD. The discovery of urea stibamine. Revista da Sociedade Brasileira de Medicina Tropical 19:115,1986.

12. Organización Mundial de la Salud. Lucha contra las leishmaniasis. Série de Informes Técnicos 793. 176p. Ginebra 1990. 